CAMS/03-02

\title{
Noncommutative Gravity
}

\author{
Ali H. Chamseddine * \\ Center for Advanced Mathematical Sciences (CAMS) and \\ Physics Department, American University of Beirut, Lebanon.
}

\begin{abstract}
Various approaches by the author and collaborators to define gravitational fluctuations associated with a noncommutative space are reviewed.
\end{abstract}

Geometry of a noncommutative space is defined by the data $(\mathcal{A}, H, D)$ where $\mathcal{A}$ is a noncommutative involutive algebra, $H$ is a separable Hilbert space and $D$ a self-adjoint operator on $H$ referred to as Dirac operator [1. Geometry on Riemannian manifolds could be recovered by specializing to the data

$$
\mathcal{A}=C^{\infty}(M), \quad H=L^{2}(S), \quad D=\gamma^{\mu}\left(\partial_{\mu}+\frac{1}{4} \omega_{\mu}^{a b} \gamma_{a b}\right),
$$

where $\omega_{\mu}^{a b}$ is the spin-connection on a manifold $M$. To deserve the name geometry the operator $D$ should satisfy certain conditions [2].

${ }^{*}$ Presented at TH-2002, Paris, France, July 2002. 
At present there are only few noncommutative spaces which are well understood such as the noncommutative space of the standard model, the noncommutative torus, deformed plane $R_{\theta}^{n}$ and the noncommutative spheres $S^{2}$, $S^{3}, S^{4}$. It is relatively easy to develop gauge theories on noncommutative spaces. To do this we first define the one-form

$$
\rho=\sum_{i} a^{i} d b^{i}, \quad a^{i}, b^{i} \in \mathcal{A},
$$

then define an involutive representation $\pi$ of $\mathcal{A}$ on $H$ such that

$$
\pi\left(\sum_{i} a_{0} d a_{1}^{i} \cdots d a_{n}^{i}\right)=\sum_{i} a_{0}^{i}\left[D, a_{1}^{i}\right] \cdots\left[D, a_{n}^{i}\right] .
$$

The curvature is defined by $\theta=d \rho+\rho^{2}$ and integration by

$$
\int \alpha=\operatorname{Tr}_{\omega}\left(\pi(\alpha)|D|^{-d}\right)
$$

where $\operatorname{Tr}_{\omega}$ is the Dixmier trace and $d$ is defined by the condition $\operatorname{tr}_{H}\left(D^{2}+1\right)^{-p}<$ $\infty, \forall p>\frac{d}{2}$.

For gauge theories we consider $\alpha=\theta^{2}$. On a commutative space one gets, in $d=4$, the action

$$
\int d^{4} x \operatorname{Tr}\left(F_{\mu \nu} F^{\mu \nu}\right)
$$

On the noncommutative torus [3] the triplet is taken to be $(l(\mathcal{A}), l(H), D)$, where $l$ is the left-twisting operator satisfying $l(a . b)=l(a) * l(b)$ [4. The star product is defined by

$$
f * g=\left.e^{\frac{i}{2} \theta^{\mu \nu} \frac{\partial}{\partial \xi^{\mu}} \frac{\partial}{\partial \eta^{\nu}}} f(x+\xi) g(x+\eta)\right|_{\xi=\eta=0} .
$$

Then one gets [5]

$$
\int d^{4} x \operatorname{Tr}\left(F_{\mu \nu} * F^{\mu \nu}\right)
$$

where $F_{\mu \nu}=\partial_{\mu} A_{\nu}-\partial_{\nu} A_{\mu}+A_{\mu} * A_{\nu}-A_{\nu} * A_{\mu}$.

The operator $D$ includes the metric properties on the space. One can extract dynamics of fluctuations of the metric by one of two possibilities. The first is by using the spectral action principle which states that the physical action depends on the spectrum of $D[6]$. Good tests of this principle can be 
made by considering the standard model of particle physics and loop space of superstrings. As an example consider the noncommutative space of the spectral model defined by

$$
\mathcal{A}=\mathcal{A}_{1} \otimes \mathcal{A}_{2}, \quad H=H_{1} \otimes H_{2}, \quad D=D_{1} \otimes 1+\gamma_{5} \otimes D_{2},
$$

where $\left(\mathcal{A}_{1}, H_{1}, D_{1}\right)$ is the triple associated with the Riemannian manifold $M$, $\left(\mathcal{A}_{2}, H_{2}, D_{2}\right)$ is the triple associated with the discrete space

$$
\mathcal{A}_{2}=\mathbb{C} \oplus \mathbb{M}_{2}(\mathbb{C}) \oplus \mathbb{M}_{3}(\mathbb{C}),
$$

$H_{2}$ is enumerated by quarks and leptons and $D_{2}$ contains information about the Yukawa couplings. The operator $D$ satisfies the property that if $\psi \in H$ then the fermionic action will be given by

$$
I_{f}=(\psi, D \psi)
$$

which includes all fermionic interaction terms. The bosonic action is then given by

$$
I_{b}=\operatorname{Tr}\left(F\left(\frac{D^{2}}{\Lambda^{2}}\right)\right)
$$

where $\Lambda$ is a cut-off scale. At low energies, the arbitrariness in the choice of the function $F$ would only reflect itself in having few measurable parameters. We can use heat kernel methods to evaluate the above trace. For example in $d=4$ we first write $F(P)=\sum_{s} f_{s} P^{s}$ and use the identity $\operatorname{Tr}\left(P^{-s}\right)=$ $\frac{1}{\Gamma(s)} \int_{0}^{\infty} d t t^{s-1}$ Tre $e^{-t P}$ and the expansion $\operatorname{Tr} e^{-t P} \simeq \sum_{n \geq 0} t^{\frac{n}{2}-2} \int_{M} a_{n}(x, P) d v(x)$ to show that

$$
I_{b}=f_{-2} a_{0}+f_{0} a_{2}+f_{2} a_{4}+\cdots,
$$

where the $a_{n}$ are the Seeley-deWit coefficients [7] and

$$
f_{-2}=\int_{0}^{\infty} u F(u) d u, \quad f_{0}=\int_{0}^{\infty} F(u) d u, \quad f_{2}=F^{\prime}(0), \cdots .
$$

From the structure of the quarks and leptons denoted by $Q=\left(u_{L}, d_{L}, d_{R}, u_{R}\right)$ and $L=\left(\nu_{L}, e_{L}, e_{R}\right)$ one can determine the discrete triple $\left(\mathcal{A}_{2}, H_{2}, D_{2}\right)$ that will give rise to the Higgs field. For the leptonic sector one obtains

$$
D_{L}=\left(\begin{array}{cc}
\gamma^{\mu}\left(D_{\mu}-\frac{i}{2} g_{2} A_{\mu}^{\alpha} \sigma^{\alpha}+\frac{i}{2} g_{1} B_{\mu}\right) & \gamma_{5} k^{e} H \\
\gamma_{5} k^{* e} H^{\dagger} & \gamma^{\mu}\left(D_{\mu}+i g_{1} B_{\mu}\right)
\end{array}\right)
$$


and a similar expression for the Dirac operator of the quarks sector. The bosonic action is then given by

$$
\begin{aligned}
I_{b}=\int d^{4} x \sqrt{g}\left(a \Lambda^{4}+b \Lambda^{2}\left(\frac{5}{4} R-2 y^{2} H^{\dagger} H\right)\right. \\
+c\left(-18 C_{\mu \nu \rho \sigma} C^{\mu \nu \rho \sigma}+3 y^{2}\left(D_{\mu} H^{\dagger} D^{\mu} H-\frac{1}{6} R H^{\dagger} H\right)+\frac{5}{3} g_{1}^{2} B_{\mu \nu} B^{\mu \nu}\right. \\
\left.\left.\quad+g_{2}^{2} F_{\mu \nu}^{\alpha} F^{\alpha \mu \nu}+g_{3}^{2} G_{\mu \nu}^{i} G^{i \mu \nu}+3 z^{2}\left(H^{\dagger} H\right)^{2}\right)\right)+O\left(\frac{1}{\Lambda^{2}}\right)
\end{aligned}
$$

where $a, b, c$ are linearly related to $f_{-2}, f_{0}, f_{2}$ respectively, and $y$ and $z$ are functions of the Yukawa couplings. By normalizing the kinetic energies of the gauge fields, one obtains a relation between the gauge coupling constants of $S U(3), S U(2)$ and $U(1)$ which is the same as that of $S U(5)$, mainly that $g_{3}^{2}=g_{2}^{2}=\frac{5}{3} g_{1}^{2}$. After rescaling the Higgs field one gets a relation for the Higgs coupling $\lambda=\frac{4 z^{2}}{3 y^{4}} g_{3}^{2}$. Using the fact that the dominant Yukawa coupling is that of the top quark this relation simplifies to $\lambda(\Lambda)=\frac{16 \pi}{3} \alpha_{3}(\Lambda)$. Combining this relation with the renormalization group equations one obtains the bound on the Higgs mass $160 \mathrm{Gev}<\mathrm{m}_{H}<200 \mathrm{Gev}$. The unification of the couplings also implies that $\Lambda \simeq 10^{15} \mathrm{Gev}$. The spectral action thus unifies gravity with gauge and Higgs interactions.

The second possibility is to study the gravitational field of a noncommutative space from the structure of the spectral triple by defining the analogue of Riemannian geometry to be called noncommutative Riemannian geometry [1]. For this one must define connections, curvature, torsion, etc. For example if $E^{A}$ are basis and $\nabla$ a connection then we have

$$
\begin{aligned}
\nabla E^{A} & =-\Omega_{B}^{A} \otimes E^{B}, \quad T(\nabla) E^{A}=T^{A}, \\
R(\nabla) E^{A} & =-\nabla^{2} E^{A}=R_{B}^{A} \otimes E^{B},
\end{aligned}
$$

which in component form gives

$$
T^{A}=d E^{A}+\Omega_{B}^{A} E^{B}, \quad R_{B}^{A}=d \Omega_{B}^{A}+\Omega_{C}^{A} \Omega_{B}^{C} .
$$

Applying these definitions to a product of a discrete two point space times a Riemannian manifold $M$ one finds that the basis is given by $E^{a}=\left(\begin{array}{cc}\gamma^{a} & 0 \\ 0 & \gamma^{a}\end{array}\right)$ 
and $E^{5}=\left(\begin{array}{cc}0 & \gamma^{5} \\ -\gamma^{5} & 0\end{array}\right)$ and the Dirac operator by

$$
D=\left(\begin{array}{cc}
\gamma^{a} e_{a}^{\mu} D_{\mu} & \gamma_{5} \phi \\
\gamma_{5} \phi & \gamma^{a} e_{a}^{\mu} D_{\mu}
\end{array}\right)
$$

The noncommutative Einstein-Hilbert action is

$$
\begin{aligned}
I & =\left\langle E_{A}, R_{B}^{A} E^{B}\right\rangle \\
& =2 \int_{M} d^{4} x \sqrt{g}\left(R-2 \partial_{\mu} \sigma \partial_{\nu} \sigma g^{\mu \nu}\right),
\end{aligned}
$$

where $\phi=e^{-\sigma}$. To this we can add a cosmological constant $\Lambda\langle 1\rangle=\Lambda \int d^{4} x \sqrt{g}$ and allow matrix algebras for the discrete space. This would give rise to gauge fields with curvature $\theta=d \rho+\rho^{2}$, where $\pi(\rho)=\left(\begin{array}{cc}\gamma^{\mu} A_{\mu} & \gamma_{5} \phi H \\ \gamma_{5} \phi H^{\dagger} & \gamma^{\mu} A_{\mu}\end{array}\right)$. The contribution of the gauge fields to the action is then 8

$$
\begin{aligned}
I=\int_{M} d^{4} x & \sqrt{g}\left(-\frac{1}{4} \operatorname{Tr}\left(F_{\mu \nu} F^{\mu \nu}\right)-\frac{\lambda}{4 !}\left(H^{\dagger} H-m^{2} e^{-2 \sigma}\right)^{2}\right. \\
& +D_{\mu} H^{\dagger} D^{\mu} H+\left(\frac{1}{2}+k^{2} H^{\dagger} H\right) \partial_{\mu} \sigma \partial_{\nu} \sigma g^{\mu \nu} \\
& \left.+R-2 \Lambda+\partial_{\mu} \sigma \partial_{\nu}\left(H^{\dagger} H\right)\right)
\end{aligned}
$$

which is the same at the Randall-Sundrum model [9] of $4+1$ dimensional space with four-dimensional brane boundaries.

Allowing the Dirac operator $D$ to fluctuate on noncommutative spaces based on deformed spheres or deformed $R^{n}$ poses a challenge. The operator $D$ is not arbitrary, and the interesting problem to solve is to find whether there are gravitational fluctuations. The presence of a constant background B-field for D-branes leads to noncommutativity of space-time coordinates which could be realized by deforming the algebra of functions on the world volume. There are indications that the gravitational action on noncommutative branes in presence of constant background B-field is non covariant [10].

Deformed gravity could be constructed by using the spectral action if one knows the form of the deformed Dirac operator $\widetilde{D}$. At present this is not known, and all one can do is to probe for possibilities. If one assumes a 
constant background B-field then the commutator of space-time coordinates gives

$$
\left[x^{\mu}, x^{\nu}\right]=i \theta^{\mu \nu}
$$

where $\left\langle B_{\mu \nu}\right\rangle=\theta_{\mu \nu}$ and $\theta^{\mu \rho} \theta_{\rho \nu}=\delta_{\nu}^{\mu}$. In this case the vielbein would be deformed to the form

$$
e_{\mu}^{a}(x, \theta)=e_{\mu}^{a}(x)+i \theta^{\nu \rho} e_{\mu \nu \rho}^{a}(x)+\cdots
$$

the complex part is dependent on $\theta$. If a metric is defined by $g_{\mu \nu}=e_{\mu}^{a} * e_{\nu a}$ then the metric will be complex. We can write $g_{\mu \nu}=G_{\mu \nu}+i B_{\mu \nu}$ and then impose the hermiticity of $g_{\mu \nu}$ which implies that $G_{\mu \nu}$ is a symmetric tensor and $B_{\mu \nu}$ is antisymmetric tensor. This leads to complex gravity. At the linearized level, the field $B_{\mu \nu}$ has the correct kinetic terms, but the ghost modes present in this field propagate at the non-linear level. The reason for the inconsistency is that there is no symmetry similar to diffeomorphism invariance associated with the field $B_{\mu \nu}$ [12].

An alternative approach is to develop a gauge theory for the field $e_{\mu}^{a}$ and formulate gravity as a noncommutative gauge theory without using a metric. This can be done without much complications in four-dimensions. The procedure is generalizable to higher dimensions, but the analysis will be more complicated. The idea is to start from the gauge group $U(2,2)$ in four-dimensions. The gauge field is expanded in the form 13

$$
A_{\mu}=i a_{\mu}+b_{\mu} \Gamma_{5}+e_{\mu}^{a} \Gamma_{a}+f_{\mu}^{a} \Gamma_{a} \Gamma_{5}+\frac{1}{4} \omega_{\mu}^{a b} \Gamma_{a b} .
$$

The gauge field strength is given by $F=d A+A^{2}$ where we have defined $A=A_{\mu} d x^{\mu}$ and $F=\frac{1}{2} F_{\mu \nu} d x^{\mu} \wedge d x^{\nu}$. To make the system dynamical without using a metric a constraint is imposed. It is given by

$$
F_{\mu \nu}^{a}+F_{\mu \nu}^{a 5}=0 \text {. }
$$

This breaks the symmetry to $S L(2 \mathbb{C})$, which is the relevant symmetry for gravity. The constraint is solved by

$$
f_{\mu}^{a}=\alpha e_{\mu}^{a}, \quad \omega_{\mu}^{a b}=\omega_{\mu}^{a b}\left(e_{\mu}^{a}+f_{\mu}^{a}\right), \quad b_{\mu}=0 .
$$

An action which is invariant under the surviving $S L(2 \mathbb{C})$ is given by

$$
\begin{aligned}
I & =i \int \operatorname{Tr}\left(\Gamma_{5} F \wedge F\right) \\
& =\frac{i}{4} \int d^{4} x \epsilon^{\mu \nu \rho \sigma} \epsilon_{a b c d}\left(R_{\mu \nu}^{a b}+8\left(1-\alpha^{2}\right) e_{\mu}^{a} e_{\nu}^{b}\right)\left(R_{\rho \sigma}^{c d}+8\left(1-\alpha^{2}\right) e_{\rho}^{c} e_{\sigma}^{d}\right) .
\end{aligned}
$$


This action consists of a Gauss-Bonnet topological term, an Einstein term and a cosmological term, provided that $\alpha \neq 1$. The advantage of this formulation is that it immediately generalizes to a noncommutative action as the metric is not used in the construction, but is generated as a function of $e_{\mu}^{a}$. Let $\widetilde{A}=\widetilde{A}_{\mu}^{I} T_{I} d x^{\mu}$ be the gauge field over the noncommutative space where $T_{I}$ are the group generators. The curvature is then given by $\widetilde{F}=d \widetilde{A}+\widetilde{A} * \widetilde{A}$. Notice that we can write

$$
\widetilde{A} * \widetilde{A}=\frac{1}{2}\left(\widetilde{A}_{\mu}^{I} *_{s} \widetilde{A}_{\nu}^{J}\left[T_{I}, T_{J}\right]+\widetilde{A}_{\mu}^{I} *_{a} \widetilde{A}_{\nu}^{J}\left\{T_{I}, T_{J}\right\}\right) d x^{\mu} \wedge d x^{\nu},
$$

where the symmetric and antisymmetric star products are defined to be, respectively, even and odd functions of $\theta$. Notice that this is consistent with imposing the constraint

$$
\widetilde{F}_{\mu \nu}^{a}+\widetilde{F}_{\mu \nu}^{a 5}=0
$$

because these correspond to generators with an odd number of gamma matrices and preserve the subgroup $S L(2 \mathbb{C})$. The noncommutative action is then given by

$$
\begin{aligned}
I & =i \int \operatorname{Tr}\left(\Gamma_{5} F * F\right) \\
& =i \int d^{4} x \epsilon^{\mu \nu \rho \sigma}\left(2 \widetilde{F}_{\mu \nu}^{1} *_{s} \widetilde{F}_{\rho \sigma}^{5}+\epsilon_{a b c d} \widetilde{F}_{\mu \nu}^{a b} *_{s} \widetilde{F}_{\rho \sigma}^{c d}\right)
\end{aligned}
$$

The constraints could be solved by using the Seiberg-Witten map [5] which enables us to express all the deformed fields in terms of the undeformed ones. The final result is 13

$$
I=i \int d^{4} x \epsilon^{\mu \nu \lambda \sigma}\left(\epsilon_{a b c d} F_{\mu \nu}^{a b} F_{\lambda \sigma}^{c d}+\theta^{\kappa \rho}\left(2 e_{\mu}^{a+} e_{\nu a}^{-} F_{\lambda \sigma \kappa \rho}^{1}+\epsilon_{a b c d} F_{\mu \nu}^{a b} F_{\lambda \sigma \kappa \rho}^{c d}\right)\right)+O\left(\theta^{2}\right)
$$

where $F_{\lambda \sigma \kappa \rho}^{c d}$ is the deformation to first order in $\theta$ of $F_{\lambda \sigma}^{c d}$.

This deformed action gives deviations from the Einstein-action which are evaluated explicitly. In this deformation there are no additional propagating degrees of freedom, all new terms being functions of the vierbein $e_{\mu}^{a}$ and its derivatives. This suggests that there should exist a formulation of noncommutative gravity on deformed $R_{\theta}^{n}$ obtained by allowing fluctuations to the Dirac operator in the spectral triple of this space. The nature of such extension is presently under investigation. 
To conclude, it is clear from the above discussion that gauge theories on noncommutative spaces are straightforward, but to define the gravitational action on such spaces is more difficult. At present there are only partial answers, and more intensive research in this direction is needed.

\section{References}

[1] A. Connes, "Noncommutative Geometry", Academic Press, New York, 1994.

[2] A. Connes, J. Math. Phys. 36 (1995) 6194.

[3] A. Connes, M. R. Douglas and A. Schwartz, JHEP 9802 (1998) 003.

[4] A. Connes and G. Landi, math.QA/0011194.

[5] N. Seiberg and E. Witten, JHEP 9909 (1999) 032.

[6] A. H. Chamseddine and A. Connes, Comm. Math. Phys. 186 (1997) 731. For details see D. Kastler, J.Math. Phys. 46 (2000) 3867.

[7] P. Gilkey, "Invariance Theory, the heat equation and the Atiyah-Singer Index Theorem", Dilmington, Publish or Perish, 1984.

[8] F. Lizzi, G. Mangano and G. Miele, Mod. Phys. Lett. A16 (2001) 1.

[9] L. Randall and R. Sundrum, Phys. Rev. Lett. 83 (1999) 3370.

[10] F. Ardalan, H. Arfai, M. R. Garousi and A. Ghodsi, hep-th/0204117

[11] A. H. Chamseddine, G. Felder and J. Fröhlich, Comm. Math. Phys. 155 (1993) 205; A. H. Chamseddine J. Fröhlich and O. Grandjean, J. Math. Phys. 36 (1995) 6255.

[12] A. H. Chamseddine, Comm. Math. Phys. 218 (2001) 283.

[13] A. H. Chamseddine, hep-th/0202137.

[14] A. Connes and M. Dubois-Violette, math-qa/0206205. 